\title{
SELETIVIDADE DE CARFENTRAZONE-ETHYL AOS MILHOS DOCE E NORMAL
}

\author{
DÉCIO KARAM ${ }^{1}$, JOSÉ FRANCISCO R. LARA², PAULO CÉSAR MAGALHÃES ${ }^{1}$, ISRAEL \\ ALEXANDRE PEREIRA FILHO ${ }^{1}$, MICHELLE B. CRUZ ${ }^{3}$.
}

\author{
${ }^{1}$ Pesquisadores, Embrapa Milho e Sorgo. Rod. MG 424, Caixa Postal 151, CEP. 35701-970 Sete Lagoas, MG. E-mail: \\ karam@cnpms.embrapa.br (autor para correspondência) \\ ${ }^{2}$ EPAMIG, Sete Lagoas, $M G$. \\ ${ }^{3}$ Graduanda em Ciências Biológicas - Unicentro Izabela Hendrix, Belo Horizonte, MG.
}

Revista Brasileira de Milho e Sorgo, v.3, n.1, p.62-68, 2004

\begin{abstract}
RESUMO - Com o objetivo de avaliar a seletividade de carfentrazone-ethyl, herbicida pósemergente com ação de inibição da enzima protoporfirinogeneo oxidase (PROTOX), sobre cultivares de milho normal e doce, foram instalados experimentos sob condições de casa de vegetação, na Embrapa Milho e Sorgo, em Sete Lagoas, MG. Os experimentos foram dispostos em blocos casualizados, com quatro repetições. Foram realizadas avaliações visuais de fitotoxicidade aos 7 e 14 dias após a aplicação (DAA) do herbicida e acúmulo de matéria seca aérea, aos 14 DAA. No primeiro ensaio, utilizaram-se cinco cultivares de milho normal (SHX 4001; AS 1533; DKB 747; 30 F 98; CD 302;), cinco de milho doce (HT - 1; BR 400; $\mathrm{HT}-3$; BR 401; BR 402) e duas doses de carfentazone-ethyl (12,5 e 25,0 $\left.\mathrm{g} \mathrm{ha}^{-1}\right)$. O maior índice de fitotoxicidade observado não ultrapassou $20 \%$, sendo as cultivares $30 \mathrm{~F} 98$ e CD 302 as mais sensíveis. Não foi detectada diferença significativa para o acúmulo de matéria seca aérea. Em outro ensaio, foi avaliado o efeito de doses de carfentrazone-ethyl $(3,13 ; 6,25$; 12,$\left.5 ; 25,0 ; 50,0 ; 100,0 ; 200,0 \mathrm{~g} \mathrm{ha}^{-1}\right)$ na cultivar BRS 3060. Nas avaliações de fitotoxicidade, aos 7 e 14 DAA, e para o acúmulo de matéria seca aérea, aos 14 DAA, foram realizadas análises de regressão. Todas as regressões apresentaram coeficientes de determinação superiores a 0,74 , demonstrando haver boa correlação entre as doses e os parâmetros analisados. Os maiores índices fitotóxicos não ultrapassaram 40\%, independente da avaliação realizada. Carfentrazone-ethyl a $50 \mathrm{~g} \mathrm{ha}^{-1}$ reduziu o acúmulo de matéria seca aérea em aproximadamente $7 \%$. Pode-se concluir que carfentrazone-ethyl apresenta boa seletividade para as cultivares estudadas, podendo ser mais uma opção para controle de plantas daninhas na cultura do milho.
\end{abstract}

Palavras-chave: fitotoxicidade, herbicida, $\mathrm{LD}_{50}$, dose resposta, Zea mays

\section{CARFENTRAZONE-ETHYL SELECTIVITY FOR NORMAL AND SWEET CORN}

\begin{abstract}
The objective of this work was to evaluate the selectivity of carfentrazoneethyl, a post emergence herbicide that inhibits a protoporphyrinogen oxidase (Protox) for normal and sweet corn. Experiments were set up at Embrapa Milho e Sorgo, Sete Lagoas, MG under greenhouse conditions. The experiments were carried out in a completely randomized block design with four replications. Evaluations of visual injury symptoms were done at 7 and 14 days after herbicide application (DAA), and of shoot dry matter at 14 DAA. In the first experiment, five normal corn cultivars (SHX 4001, AS 1533, DKB 747, 30 F 98, CD 302), five sweet corn cultivars (HT 1, BR 400, HT 3, BR 401, BR 402), and two carfentrazone-ethyl
\end{abstract}


rates ( 12.5 and $25.0 \mathrm{~g} \mathrm{ha}^{-1}$ ) were tested. The injury level observed was not greater than $20 \%$. The cultivars $30 \mathrm{~F} 98$ and CD 302 were the most susceptible ones. It was not detected any significant differences to shoot dry matter. In another experiment, the response of the cultivar BRS 3060 to carfentrazone rates $\left(3.13 ; 6.25 ; 12.5 ; 25.0 ; 50.0 ; 100.0 ; 200.0 \mathrm{~g} \mathrm{ha}^{-1}\right)$ was tested. Regression analyses were done considering injury levels (7 and 14 DAA) and shoot dry weight (14 DAA) against carfentrazone-ethyl rates. The coefficient of determination of the regressions was higher than 0,74 , showing good relationship between rates and injury level or shoot dry weight. The injury level observed was not higher than $40 \%$. Carfentrazone-ethyl at $50 \mathrm{~g} \mathrm{ha}^{-1}$ reduced about $7 \%$ of the shoot dry weight. The results indicate that carfentrazoneethyl have acceptable selectivity to control weeds on corn.

Key words: phytotoxicity, herbicide, $\mathrm{LD}_{50}$, dose response, Zea mays

O milho, um dos cereais mais plantados no Brasil, apresentou produtividade média de $3.128 \mathrm{~kg}$ ha $^{-1}$ na $1^{\text {a }}$ safra (verão), em $2001 / 02$, e de $2.153 \mathrm{~kg}$ ha ${ }^{-1}$ na $2^{\mathrm{a}}$ safra (safrinha), em 2002, produtividades estas consideradas baixas (Conab, 2003). Entre os fatores redutores de produtividade, destacam-se as plantas daninhas, que são responsáveis por perdas que variam de $12 \%$ a $32 \%$ no rendimento da cultura (Cruz \& Ramalho, 1983; Rossi et al., 1996), podendo alcançar índices superiores a 75\%, quando em convivência com 160 plantas de Brachiaria plantaginea por $\mathrm{m}^{2}$ (Spader \& Vidal, 2000).

O período crítico de competição, ou seja, quando os efeitos das plantas infestantes efetivamente causam prejuízo à cultura, é variável em função de alguns fatores, tais como a espécie e densidade das plantas infestantes, o genótipo da cultura e as condições ambientais. Para a cultura do milho, o período de competição tem sido relatado entre 15 e 56 dias (Hall et al., 1992).

Entre as alternativas de controle das plantas infestantes na cultura do milho, o uso de herbicidas seletivos tem-se destacado nos últimos anos. Entretanto, o uso indiscriminado dos mesmos tem ocasionado efeitos indesejáveis, como a fitotoxicidade à cultura. A seletividade de espécies vegetais depende da capacidade de tolerância destas aos herbicidas, sendo que essa tolerância baseia-se na capacidade de degradação ou metabolização dos herbicidas em produtos não tóxicos nas plantas. No entanto, muitas vezes, essa tolerância pode variar em função da dose do herbicida e das condições do ambiente (Radosevich et al., 1997).

Entre os herbicidas registrados para a cultura do milho encontra-se o carfentrazone-ethyl, pertencente ao grupo químico das aril-triazolinonas, classificado como pós-emergente. Seu modo de ação consiste na inibição da enzima protoporfirinogênio oxidase (PROTOX), envolvida na biossíntese da clorofila, resultando no acúmulo de protoporfirinogênio IX (PPIX) no citoplasma (Sherman et al., 1991; Dayan et al., 1997). Na presença de luz, PPIX forma oxigênio singleto, que é responsável pela morte das plantas através da peroxidação das membranas (Devine et al., 1993). Devido a essa ação, os sintomas de fitotoxicidade podem ser observados dentro de poucas horas após a aplicação, sendo a morte da planta constatada em uma semana (Daylan et al., 1997).

A seletividade detectada nas plantas devido à aplicação de carfentrazone-ethyl deve-se ao metabolismo de detoxificação desse composto químico (Daylan et al., 1997). Poucos são os trabalhos encontrados na literatura sobre a seletividade de carfentrazone-ethyl à cultura do milho. Entretanto, alguns estudos demonstrando a tolerância diferenciada dessa cultura a diferentes herbicidas foram publicados por Damião Filho et al. (1996), Green (1998) e Pereira Filho et al. (2000). 
Diante da carência de informações sobre a seletividade de carfentrazone-ethyl sobre a cultura de milho, realizou-se este trabalho, na Embrapa Milho e Sorgo, em Sete Lagoas, MG, visando avaliar o efeito da aplicação de diferentes doses de carfentrazone-ethyl em cinco cultivares de milho doce e cinco de milho normal.

\section{Material e Métodos}

Experimentos foram conduzidos em condições de casa de vegetação, na Embrapa Milho e Sorgo, em Sete Lagoas, MG. Vasos com capacidade de 1,0L foram preenchidos com terra proveniente da camada superficial de um Latossolo Vermelho distrófico, textura argilosa (LVd). A adubação foi realizada baseando-se em análise do solo. $\mathrm{O}$ delineamento experimental foi o de blocos ao acaso, com quatro repetições, sendo que cada vaso contendo uma planta de milho foi considerado como uma unidade experimental. Os herbicidas foram aplicados através de pulverizador pressurizado a $\mathrm{CO}_{2}$, operando à pressão constante de $40 \mathrm{lb} \mathrm{pol}^{-2}$, munido de bico Teejet 110-02 e consumo de calda de $180 \mathrm{~L} \mathrm{ha}^{-1}$. Avaliações visuais de fitotoxicidade foram realizadas tomando-se como referência a escala de 0 (nenhum sintoma) a $100 \%$ (morte ou dano total das plantas). Análise de variância e teste de Tukey a 5\% para comparação de médias foram realizadas de acordo com Steel et al. (1997). Análises de regressão foram realizadas para interpretação do efeito de doses de carfentrazone-ethyl, utilizandose a equação hiperbólica descrita a seguir:

$Y=(A * D) /(B+D)$

em que $\boldsymbol{Y}$ é a resposta ou efeito do tratamento herbicida, $\boldsymbol{A}$ e $\boldsymbol{B}$ são coeficientes da equação e $\boldsymbol{D}$ corresponde à dose de carfentrazone-ethyl.

\section{Seletividade}

Foram instalados dois experimentos, nas datas de 02 e 14 de janeiro de 2002, onde foram utilizadas cinco cultivares de milho doce e cinco de milho normal, conforme pode ser verificado na Tabela 1. Os tratamentos foram dispostos em esquema fatorial $10 \times 2$, sendo dez cultivares e duas doses de carfentrazone-ethyl. As cultivares foram pulverizadas com o herbicida nas doses de 12,5 e 25,0g ha ${ }^{-1}$. A interação das cultivares com as doses do herbicida foram comparadas com plantas de milho não pulverizadas (testemunha). As avaliações de fitotoxicidade foram realizadas aos 7 e 14 dias após a aplicação (DAA). Aos 14 DAA, as plantas de milho foram cortadas rente ao solo, colocadas para secar em estufa de circulação forçada a $65^{\circ} \mathrm{C}$ e pesadas até peso constante, para obtenção do acúmulo de matéria seca da parte aérea.

\section{Resposta à dose de carfentrazone-ethyl}

Para a obtenção da resposta de milho ao carfentrazone-ethyl, foram também instalados dois experimentos, nas datas de 18 e 25 de janeiro de 2002. Foi utilizada a cultivar de milho BRS 3060, a qual foi pulverizada com sete doses de carfentrazoneethyl $(3,13 ; 6,25 ; 12,50 ; 25,00 ; 50,00 ; 100,00$ e $\left.200,00 \mathrm{~g} \mathrm{ha}^{-1}\right)$. As avaliações de fitotoxicidade foram realizadas aos 7 e 14 DAA. Aos 14 DAA foi realizada, conforme procedimento dos experimentos de seletividade, análise do acúmulo de matéria seca da parte aérea.

TABELA 1. Cultivares de milho doce e normal utilizadas para avaliação de seletividade ao herbicida carfentrazone-ethyl. Embrapa Milho e Sorgo, Sete Lagoas, MG, 2002.

\begin{tabular}{ll}
\hline Milho Doce & Milho Normal \\
\hline HT 1 & SHX 4001 \\
BR 400 & AS 1533 \\
HT 3 & DKB 747 \\
BR 401 & 30 F 98 \\
BR 402 & CD 302 \\
\hline
\end{tabular}




\section{Resultados e Discussão}

Os resultados obtidos foram submetidos ao teste de Bartlett, que indicou diferenças entre as variâncias dos experimentos; portanto, os resultados foram analisados separadamente (Steel et al., 1997).

Os maiores índices de fitotoxicidade visual observados foram de aproximadamente $20 \%$ e $6 \%$, nos experimentos de seletividade iniciados em 2 de janeiro e repetidos em 14 de janeiro de 2002, respectivamente (Tabela 2). Pelas análises de variância individuais dos experimentos, observa-se apenas o efeito significativo para a interação dose de carfentrazone-ethyl e cultivares para os dois experimentos apenas na dose de $25 \mathrm{~g} \mathrm{ha}^{-1}$. Na dose de
$12,5 \mathrm{~g} \mathrm{ha}^{-1}$, não foi observado efeito significativo entre cultivares. Os níveis de fitotoxicidade visuais observados nas cultivares foram significativos, em ambos os experimentos, para a dose de 25,0 $\mathrm{g} \mathrm{ha}^{-1}$, aos 7 DAA, sendo esses índices reduzidos aos 14 DAA. As cultivares $30 \mathrm{~F} 98$ e CD 302 foram as mais sensíveis, diferindo significativamente em tolerância das demais avaliadas. Aos 14 DAA, não foram detectadas diferenças significativas para fitotoxicidade visual entre cultivares, sendo o maior índice $(5,1 \%)$ observado para a cultivar $30 \mathrm{~F} 98$. Verifica-se que alguns dos milhos normais apresentaram-se mais sensíveis à aplicação de carfentrazoneethyl. No experimento de 14/01, embora com índices fitotóxicos inferiores aos do experimento iniciado em

TABELA 2. Níveis fitotóxicos observados visualmente em cultivares de milho doce e normal após a aplicação de carfentrazone-ethyl. Embrapa Milho e Sorgo, Sete Lagoas, MG, 2002.

\begin{tabular}{|c|c|c|c|c|}
\hline \multirow{4}{*}{ Cultivares de milho } & \multicolumn{4}{|c|}{ Fitotoxicidade (\%) } \\
\hline & \multicolumn{2}{|c|}{$02 / 01 / 2002^{1}$} & \multicolumn{2}{|c|}{$14 / 01 / 2002$} \\
\hline & \multicolumn{4}{|c|}{$\mathbf{D A A}^{2}$} \\
\hline & 7 & 14 & 7 & 14 \\
\hline \multicolumn{5}{|l|}{ Normal } \\
\hline SHS 4001 & $6,9 \operatorname{cde}^{3}$ & $3,1 \mathrm{ab}$ & $1,5 \mathrm{~b}$ & $1,0 \quad b$ \\
\hline AS 1533 & 9,4 bc & $2,4 \quad b$ & $1,3 \quad b$ & $1,8 \quad b$ \\
\hline DK 747 & $6,3 \mathrm{de}$ & $1,1 \quad b$ & $2,1 \quad b$ & $1,4 \quad b$ \\
\hline $30 \mathrm{~F} 98$ & $18,8 \mathrm{a}$ & $5,1 \mathrm{a}$ & $1,8 \quad b$ & $1,5 \mathrm{~b}$ \\
\hline CD 302 & $16,9 \mathrm{a}$ & $2,5 \mathrm{~b}$ & $3,1 \mathrm{ab}$ & $2,3 \mathrm{ab}$ \\
\hline \multicolumn{5}{|l|}{ Doce } \\
\hline HT 1 & $10,0 \mathrm{~b}$ & $1,8 \quad \mathrm{~b}$ & $5,5 \mathrm{a}$ & $3,4 \mathrm{a}$ \\
\hline BR 400 & $5,0 \quad \mathrm{e}$ & $1,3 \mathrm{~b}$ & $2,4 \quad b$ & $2,6 \mathrm{ab}$ \\
\hline HT 3 & $10,0 \mathrm{~b}$ & $2,5 \mathrm{~b}$ & $1,5 \mathrm{~b}$ & $1,1 \quad b$ \\
\hline BR 401 & $5,0 \quad \mathrm{e}$ & $1,1 \quad b$ & $1,0 \mathrm{~b}$ & $0,3 \mathrm{~b}$ \\
\hline BR 402 & 8,1 bcd & $2,0 \quad b$ & $3,3 \mathrm{ab}$ & $2,1 \mathrm{ab}$ \\
\hline $\mathrm{F}(\%)$ & 19,41 & 7,64 & 4,45 & 4,17 \\
\hline Média cultivar & 9,6 & 2,3 & 2,4 & 1,8 \\
\hline Média milho normal & 11,7 & 2,8 & 2,0 & 1,6 \\
\hline Média milho doce & 7,6 & 1,7 & 2,7 & 1,9 \\
\hline
\end{tabular}

${ }^{1}$ Época de plantio dos experimento

${ }^{2}$ Dias após aplicação

${ }^{3}$ Médias seguidas da mesma letra, na coluna, não diferem entre si pelo teste de Tukey, a 5\% de probabilidade. 
02/01, diferenças significativas foram detectadas quando da aplicação de carfentrazone-ethyl aos diferentes milhos doce e normal. Os índices fitotóxicos observados neste experimento foram inferiores a 5,5\%, considerados não prejudiciais à cultura dos milhos doce e normal.

O acúmulo de matéria seca não foi reduzido significativamente devido ao efeito fitotóxico do herbicida carfentrazone-ethyl, tampouco foi detectada correlação significativa entre índices de fitotoxicidade avaliada aos 7 DAA e o acúmulo de matéria seca, aos 14 DAA, para ambos os experimentos.

Os sintomas visuais de fitotoxicidade observados nos experimentos podem ser visualizados na Figura 1, onde nota-se necrose nas folhas, devido à peroxidação das membranas (Devine et al., 1993). A tolerância das plantas de milho a carfentrazone-ethyl pode estar ligada à menor absorção e/ou à rápida metabolização do herbicida (Thompson \& Nissen, 2000). Por isso, não foram verificados elevados índices de injúrias nas plantas de milho. A diferença no índice de injúria causada por carfentrazone-ethyl, nos dois experimentos, pode estar associada às condições de luminosidade e irrigação dos vasos. Resultados obtidos por Thompson
\& Nissen (2002) indicam o aumento da fitotoxicidade em milho, soja e trigo devido ao sombreamento anteriormente à aplicação do herbicida e à maior disponibilidade de água no solo e, portanto, na planta.

Os resultados obtidos nos experimentos de dose resposta estão apresentados na Figura 2. A fitotoxicidade visual máxima observada não ultrapassou a $40 \%$, tanto aos 7 quanto aos 14 DAA (Figura 2). Com este resultado, pode-se inferir que, para obter índices de fitotoxicidade superiores a $50 \%$, para a cultivar BRS 3060, seria necessária a aplicação superior a $200 \mathrm{~g} \mathrm{ha}^{-1}$ de carfentrazone-ethyl. Os índices visuais de fitotoxicidade tornaram-se menos visíveis aos 14 DAA, demostrando recuperação das plantas do milho ao longo do tempo. Devese salientar que, como qualquer herbicida de contato, os sintomas fitotóxicos são agravados em função das condições ambientais no momento da aplicação, tornando-se mais agudos com altas temperatura e luminosidade (Stevan et al., 1991; Devine et al., 1993; Daylan et al., 1997).

Resultado semelhante foi observado para o acúmulo de biomassa seca da parte aérea, confirmando a necessidade de aplicações superiores a $200 \mathrm{~g} \mathrm{ha}^{-1}$ de carfentrazone-ethyl para que as plantas de milho fossem reduzidas em $50 \%$ (Figura2). A

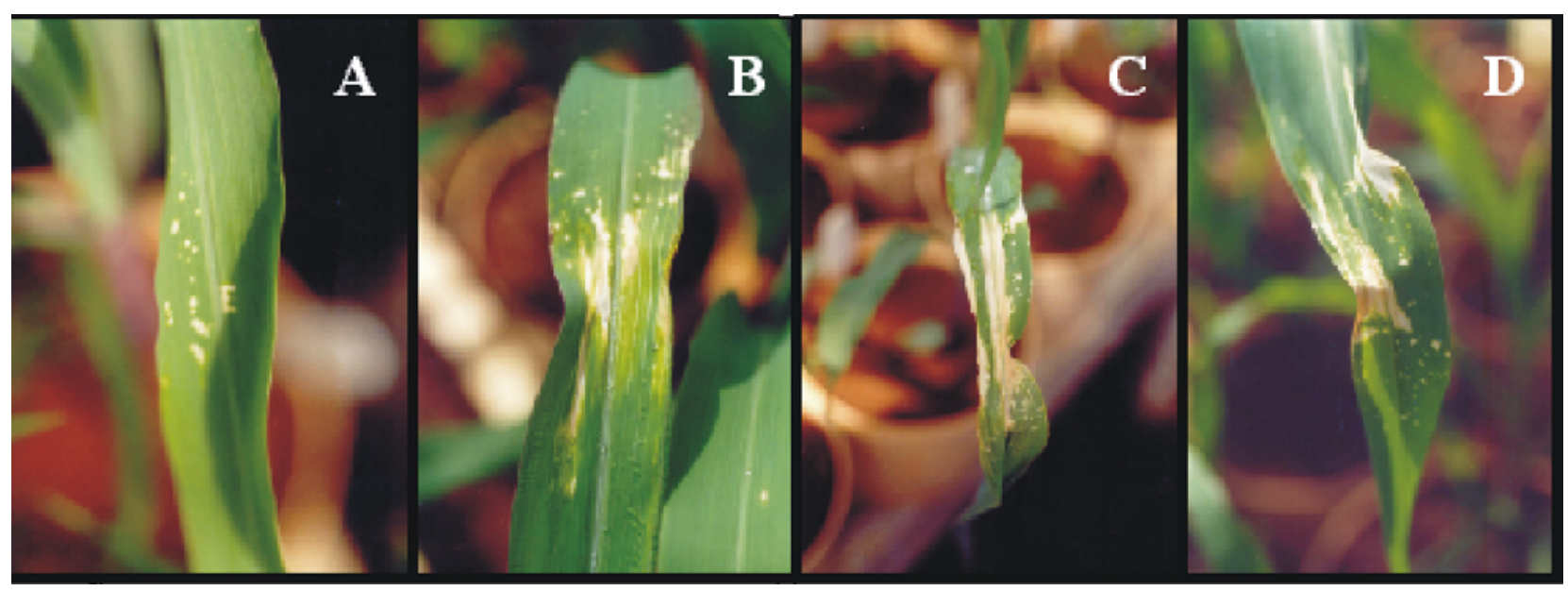

FIGURA 1. Efeito fitotóxico de carfentrazone-ethyl em plantas de milho, aos dez dias após a aplicação. Embrapa Milho e Sorgo, Sete Lagoas, MG, 2002. 

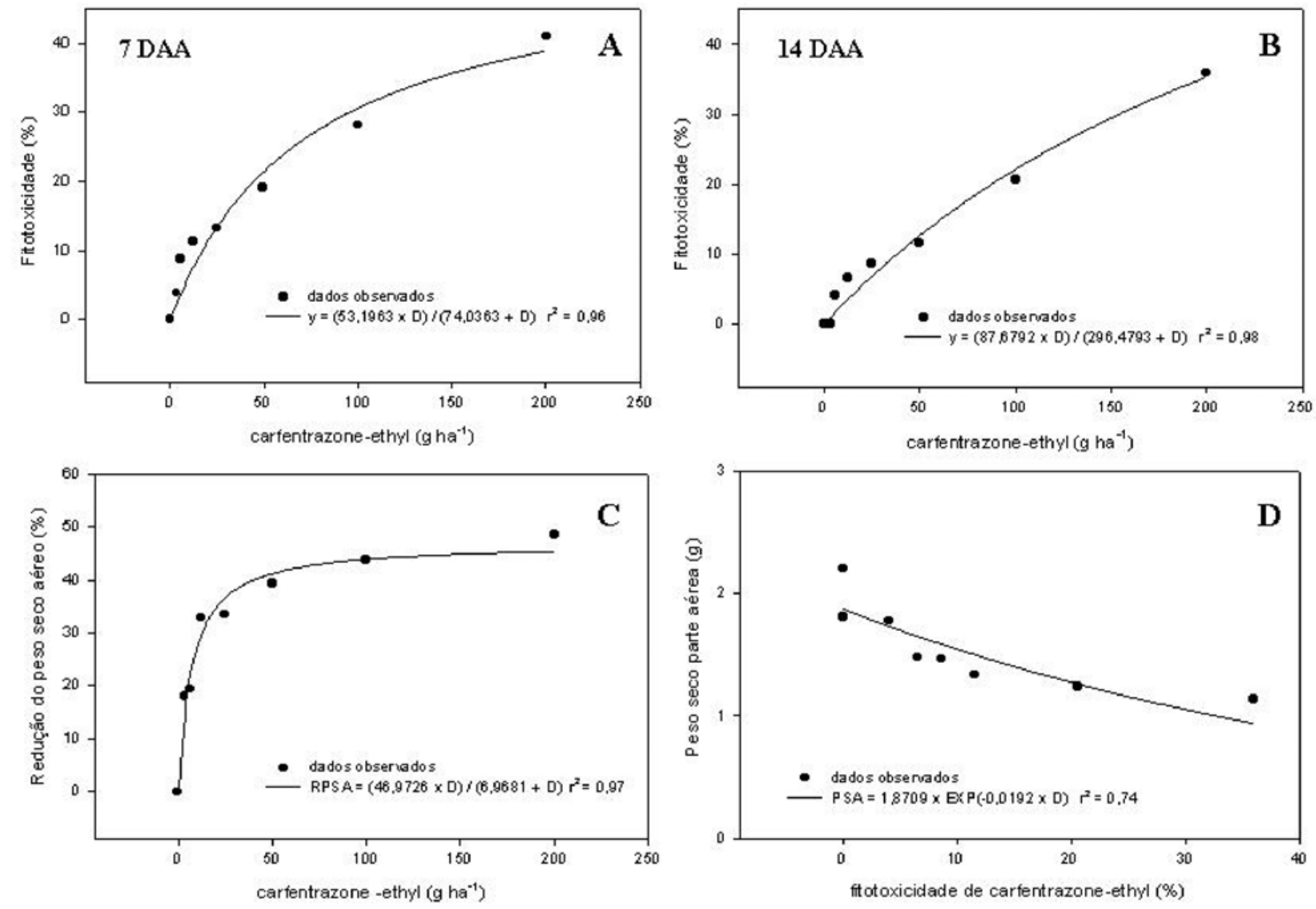

FIGURA 2. Fitotoxicidade visual de carfentrazone-ethyl aos 7 (A) e 14 (B) dias após a aplicação (DAA), redução da matéria seca aérea (C) e peso seco aéreo (D), observados na cultivar de milho BRS 3060 . Embrapa Milho e Sorgo, Sete Lagoas, MG, 2002.

dose registrada e recomendada para a cultura do milho, ou seja, 12,5 $\mathrm{g} \mathrm{ha}^{-1}$ de carfentrazone-ethyl, ocasionou reduções aos 14 dias após a aplicação da ordem de $7 \%$ na matéria seca aérea das plantas de milho BRS 3060 (Figura 2), indicando que esta cultivar apresenta boa seletividade desse herbicida para esse híbrido.

\section{Conclusões}

As cultivares de milho doce e normal são tolerantes a carfentrazone-ethyl, podendo o mesmo ser recomendado para o controle de plantas daninhas nessa cultura.

\section{Literatura Citada}

COMPANHIA NACIONAL DE ABASTECIMENTO, CONAB. Disponível em: <http:// www.conab.gov.br>. Acesso em: 5 jun. 2003.

CRUZ, J. C.; RAMALHO, M. A. P. Tração animal no controle de plantas daninhas na cultura do milho. In: EMBRAPA. Centro Nacional de Pesquisa de Milho e Sorgo. Mecanização na cultura do milho: utilização de tração animal. Sete Lagoas, 1983. p. 24 - 42. (EMBRAPA - CNPMS. Circular Técnica, 09). 
DAMIÃO FILHO, C. F.; MÔRO, F. V., TAVEIRA, L. R. Respostas de híbridos de milho ao nicosulfuron. 1 - Aspectos biológicos e da produção. Planta Daninha, Viçosa, v. 14, n. 1, p. 3-13, 1996.

DAYLAN, F. E.; DUKE, S. O.; WEETE, J. D.; HANCOCK, H. G. Selectivity and mode of action of carfentrazone-ethyl, a novel phenyl triazolinone herbicide. Pesticide Science, Oxford, v. 51, n. 1, p. 65-73 1997.

DEVINE, M. D.; DUKE, S. O.; FEDTKE, C. Oxygen toxicity and herbicidal action. In: DEVINE, M. D.; DUKE, S. O.; FEDTKE, C. Physiology of herbicide action. Englewood Cliffs: Prentice Hall, 1993. p. 177-188.

GREEN, J. M. differential tolerance of corn (Zea mays) inbreds to four sulfonyurea herbcides and bentazon. Weed Technology, Lawrence, v. 12, p. 474-477, 1998

HALL, M. R.; SWANTON, C. J.; ANDERSON, $\mathrm{G}$. W. The critical period of weed control in grain corn (Zea mays). Weed Science, Lawrence, v. 40, n. 3, p. 441-447, 1992.

PEREIRA FILHO, I. A.; OLIVEIRA, M. F.; PIRES, N. M. Tolerância de híbridos de milho ao herbicida nicosulfuron. Planta Daninha, Viçosa, v. 18, n. 3, p. 479-482, 2000.

RADOSEVICH, S.; HOLT, J.; GHERSA, C. Herbicide symptoms and selectivity. In: RADOSEVICH, S.; HOLT, J.; GHERSA, C. Weed Ecology: implications for management. New York: John Willey, 1997. p. $413-425$.
ROSSI, I. H.; OSUMA, J. A.; ALVES, P. L. C. A.; BEZUTE, A. J. Interferência das plantas daninhas sobre algumas características agronômicas e a produtividade de sete cultivares de milho. Planta Daninha, Viçosa, v. 14, n. 2, p. 134-148, 1996.

SHERMAN, T. D.; BECERRIL, J. M.; MATSUMOTO, H.; DUKE, M. V.; JACOBS, J. M.; JACOBS, N. J.; DUKE, S. O. Physiological basis for differential sensitivities of plant species to protoporphyrinogen oxidase inhibiting herbcides. Plant Physiology, Bethesda, v. 97, p. 280 - 287, 1991.

SPADER, V.; VIDAL, R. A. Interferência de Brachiaria plantaginea sobre características agronômicas, componentes do rendimento e produtividade de grãos de milho. Planta Daninha, Viçosa, v. 18 , n. 3, p. 465 - 470, 2000.

STEEL, R. G. D.; TORRIE, J. H.; DICKEY, D. A. Principles and procedures of statistics a biometric approach. New York: McGraw-Hill, 1997. $633 \mathrm{p}$.

THOMPSON, W. M.; NISSEN, S. J. Absorption and fate of carfentrazone-ethyl in Zea mays, Glycine max, and Abutilon theophrasti. Weed Science, Lawrence, v.48, n. 1, p. 15-19, 2000.

THOMPSON, W. M.; NISSEN, S. J. Influence of shade and irrigation on the response of corn (Zea mays), soybean (Glycine max), and wheat (Triticum aestivum) to carfentrazone-ethyl. Weed Science, Lawrence, v. 16, n. 2, p. 314-318, 2002. 